$1-20-2003$

\title{
Analysis of the Shadow-Sausage Effect Caustic
}

James A. Lock

Cleveland State University, j.lock@csuohio.edu

Charles L. Adler

Diana Ekelman

Jonathan Mulholland

Brian Keating

Follow this and additional works at: https://engagedscholarship.csuohio.edu/sciphysics_facpub

Part of the Physics Commons

How does access to this work benefit you? Let us know!

\section{Publisher's Statement}

This paper was published in Applied Optics and is made available as an electronic reprint with the permission of OSA. The paper can be found at the following URL on the OSA website: http://www.opticsinfobase.org/ao/abstract.cfm?URI=ao-42-3-418. Systematic or multiple reproduction or distribution to multiple locations via electronic or other means is prohibited and is subject to penalties under law.

\section{Original Citation}

Lock, James A., Charles L. Adler, Diana Ekelman, Jonathan Mulholland, and Brian Keating. "Analysis of the Shadow-Sausage Effect Caustic." Applied Optics 42 (2003): 418-428.

\section{Repository Citation}

Lock, James A.; Adler, Charles L.; Ekelman, Diana; Mulholland, Jonathan; and Keating, Brian, "Analysis of the Shadow-Sausage Effect Caustic" (2003). Physics Faculty Publications. 44.

https://engagedscholarship.csuohio.edu/sciphysics_facpub/44

This Article is brought to you for free and open access by the Physics Department at EngagedScholarship@CSU. It has been accepted for inclusion in Physics Faculty Publications by an authorized administrator of EngagedScholarship@CSU. For more information, please contact library.es@csuohio.edu. 


\title{
Analysis of the shadow-sausage effect caustic
}

\author{
James A. Lock, Charles L. Adler, Diana Ekelman, Jonathan Mulholland, and Brian Keating
}

\begin{abstract}
We analyze the optical caustic produced by light refracted at the curved meniscus surrounding a cylindrical rod standing partially out of a liquid-filled container. When the rod is tilted from the vertical or when light is diagonally incident, the caustic is a four-cusped astroid with two of its cusps obscured by the rod's shadow. If a portion of the flat end of the rod is raised above the water level, the caustic evolves into a pattern of five interlocking cusps. The five cusps result from symmetry breaking of a three-cusped surface perturbation caustic. (C) 2003 Optical Society of America

OCIS codes: 290.0290, 080.1510, 080.1010.
\end{abstract}

\section{Introduction}

The subject of this paper is the analysis of the following set of observations known as the shadow-sausage effect. $^{1-4} \quad$ A long straight tree branch of diameter $\sim 2$ $\mathrm{cm}$ stands partially out of a quiet shallow stream as in Fig. 1(a). A curved meniscus of the water surface is formed in the vicinity of its attachment to the branch. Sunlight casts a shadow of the branch on the sediments at the bottom of the stream. Because of refraction at the curved meniscus, the shadow consists of two disjoint segments corresponding to the portions of the branch above and below the water surface. The end of each shadow segment is pointed. The two shadow segments were named the shadow-sausage effect by the author of Ref. 1 , since they resembled a pair of linked sausages. This optical effect may be observed in countless other situations in which a smooth cylindrical object extends out of a liquid-filled container. For example, in Ref. 1 the effect was observed when light from an overhead fixture was refracted at the meniscus of a pencil held diagonally in a bathtub, and one of the present authors (Adler) first noticed the effect when sunlight was refracted at the meniscus of a rope hanging out of a boat partially filled with water.

J. A. Lock (j.lock@csuohio.edu) is with the Department of Physics, Cleveland State University, Cleveland, Ohio 44115. C. L. Adler, D. Ekelman, J. Mulholland, and B. Keating are with the Department of Physics, St. Mary's College of Maryland, St. Mary's City, Maryland 20686.

Received 17 December 2001; revised manuscript received 4 March 2002.

0003-6935/03/030418-11\$15.00/0

(C) 2003 Optical Society of America
Two bright cusp caustics pointing back-to-back occur in the illuminated region between the two shadow segments. If the rod lies in the plane containing the light source and the normal to the asymptotically flat water surface, the two cusps are symmetric, are of equal size, and have a common axis perpendicular to the rod's shadow as is shown in Fig. 1. The shadows of the right and left edges of the rod far above and far below the meniscus lie directly beneath the respective edges. But the left cusp caustic is continuously connected to the right shadow edge, and the right cusp is continuously connected to the left shadow edge. If the rod does not lie in the plane containing the light source and the normal to the water surface, the two cusps differ in size, they are not symmetric, and their axes are not perpendicular to the rod's shadow.

If the end of the rod has been cut reasonably flat and a small portion of the cut end is raised above the water level, the refraction caustic evolves into a pattern of five interlocking cusps as is shown in Fig. 2. Four of the cusps point in the general direction of the light source with two on one side of the rod's shadow and two on the other side. The fifth cusp points away from the light source and lies on the rod's shadow. When we vary the tilt angle of the rod or the percentage of the flat end above the water level, the pair of cusps to either side of the shadow move closer together or farther apart while the cusp on the shadow grows or shrinks in size. This progression is apparent in Figs. 2(b)-2(d).

In the observations of Figs. 1 and 2, the rod was tilted far from the vertical, sunlight was incident far from vertical, and the slope of the meniscus of the water surface at its attachment to the rod was large. These conditions make the refraction caustic difficult 


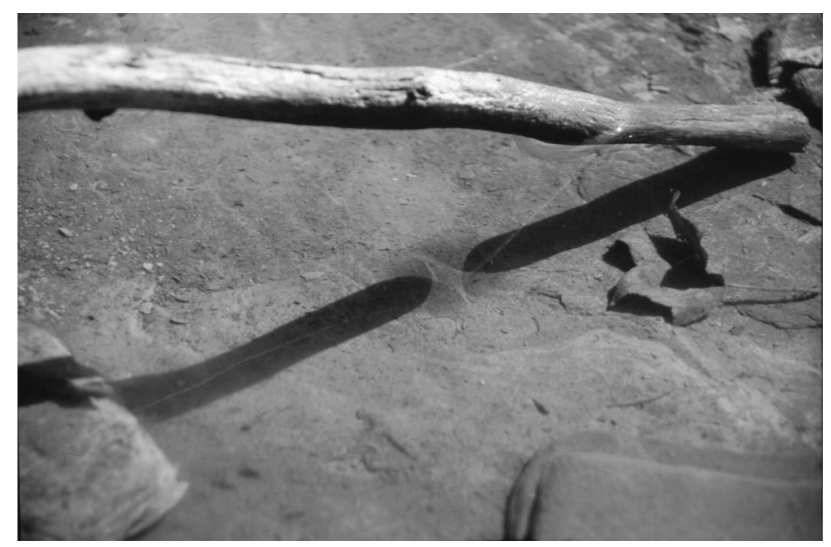

(a)

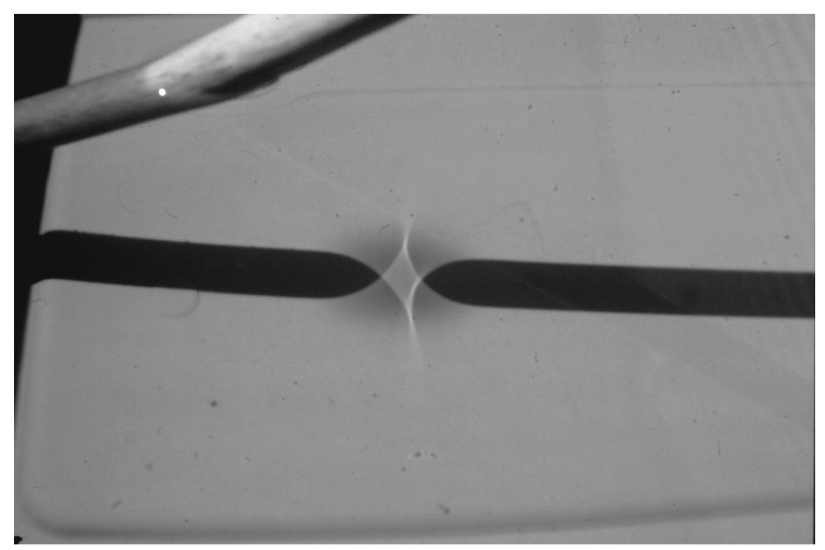

(b)

Fig. 1. Shadow-sausage effect for (a) a tree branch extending diagonally out of a shallow stream and (b) a 2.5-cm-diameter wooden dowel rod extending diagonally out of a 15-cm-deep plastic tub filled with water and illuminated by the Sun. The branch and the dowel rod lie in the plane formed by the Sun's rays and the normal to the asymptotically flat water surface, and the portion above the surface is tilted away from the Sun.

to investigate analytically. As a result, in Sections 2-6 we assume that the rod is nearly vertical, incident light propagates nearly vertically downward, the meniscus slope is everywhere small, and the radius of the rod is much larger than the scale length of the meniscus. The physical mechanism responsible for the formation of the refraction caustic under these less-severe conditions is assumed to describe qualitatively the observations of Figs. 1 and 2 as well.

The body of this paper is organized as follows. In Section 2 we determine the approximate meniscus shape for a circular cross-section rod standing either vertically or diagonally in a large container filled with a liquid under the assumption that the meniscus slope is everywhere small. In Sections 3 and 4 we determine the shape of the refraction caustic for light vertically incident on the meniscus of a vertical or a tilted rod, respectively. The circularly symmetric meniscus surrounding a vertical rod produces an axial refraction caustic whose shape is unstable with respect to perturbations in either the shape of the meniscus or the direction of the incident light. ${ }^{5}$ The refraction caustic occurring for light vertically incident on the meniscus of a tilted rod is a four-cusped astroid, one of the axial caustics studied in Ref. 6 in the context of glory scattering by a harmonically perturbed liquid droplet. In Section 5 we determine the shape of the caustic produced by light diagonally incident on the meniscus of a vertical rod. We find that the optical path length in the liquid contains terms analogous to coma and astigmatism of skew rays passing through a lens. The caustic produced by both the astigmatism term to first order and the coma term to second order are astroids whose combination agrees with the observations of Fig. 1.

When a portion of the flat end of the rod is raised above the water level, the dominant terms in the Fourier-series expansion of the meniscus shape are proportional to $\cos (\phi)$ and $\cos (3 \phi)$. The refraction caustic produced by the $\cos (3 \phi)$ term to first order has three cusps, each of which is twofold degenerate. ${ }^{6}$ The contribution to the caustic produced by the $\cos (3 \phi)$ term taken to second order and other Fourier terms such as $\cos (2 \phi)$ taken to first order break the twofold degeneracy and twin each of the three cusps in agreement with the observations of Figs. 2(a)-2(d). Finally, in Section 7 we summarize our conclusions.

\section{Shape of the Liquid Meniscus}

We consider a circular cross-section rod of radius $a$ extending out of a large container filled with a liquid as in Fig. 3. The rod's axis passes through the origin of coordinates, and the asymptotically flat liquid surface coincides with the $x y$ plane. The liquid rises above the $x y$ plane to meet the rod forming a meniscus whose height in polar coordinates is $f(r, \phi)$ with $f$ $\rightarrow 0$ as $r \rightarrow \infty$. If $C_{\text {mean }}$ is the mean curvature ${ }^{7}$ of the meniscus at any point on the liquid surface, $\gamma$ and $\rho$ are the liquid's surface tension and density, and $g$ is the Earth's gravitational acceleration, then the shape of the meniscus is the solution to the nonlinear partial differential equation ${ }^{8}$

$$
2 \mathrm{C}_{\text {mean }}-f / L^{2}=0
$$

where

$$
L \equiv(\gamma / \rho g)^{1 / 2}
$$

is the length scale of the meniscus and has the numerical value $2.73 \mathrm{~mm}$ for water. If the slope of the liquid surface in the vicinity of the rod is everywhere small, Eq. (1) simplifies to the linear partial differential equation ${ }^{9}$

$$
\nabla^{2} f-f / L^{2}=0
$$

whose solution in polar coordinates is

$$
\begin{aligned}
f(r, \phi)= & A_{0}\left[K_{0}(r / L)+\sum_{p=1}^{\infty} \epsilon_{p} K_{p}(r / L) \cos (p \phi)\right. \\
& \left.+\sum_{p=1}^{\infty} \delta_{p} K_{p}(r / L) \sin (p \phi)\right],
\end{aligned}
$$




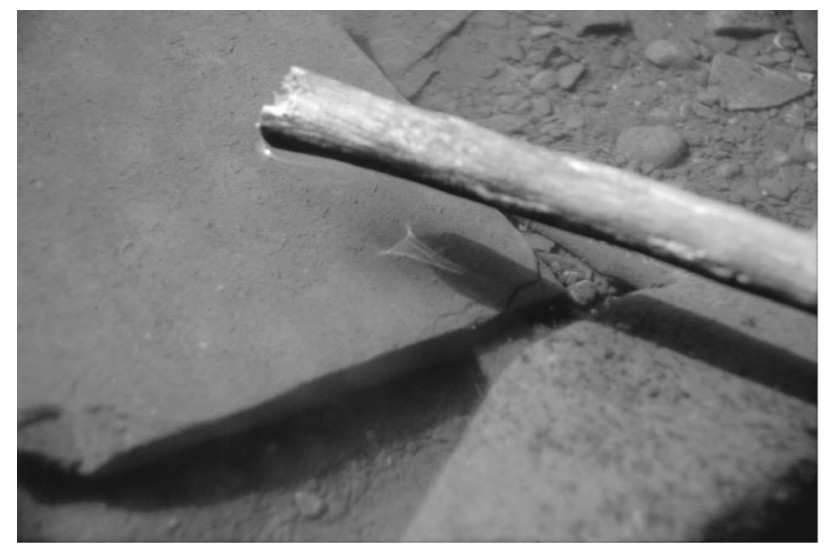

(a)

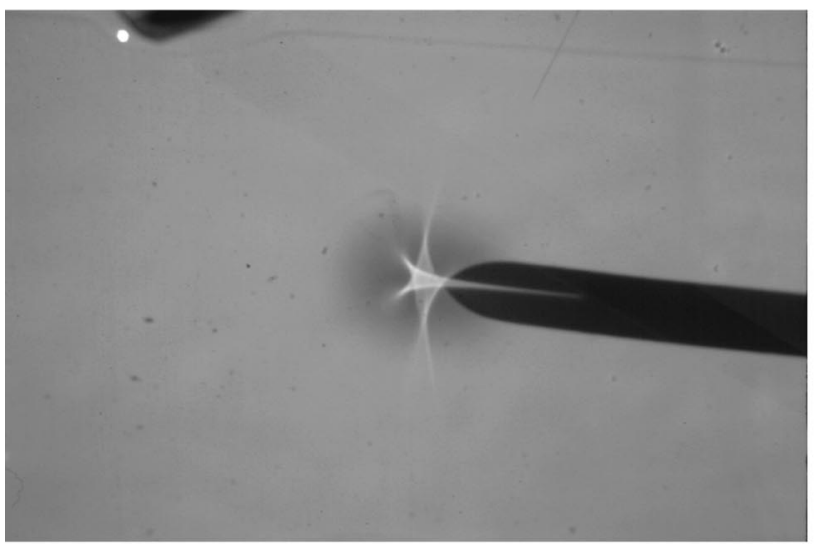

(c)

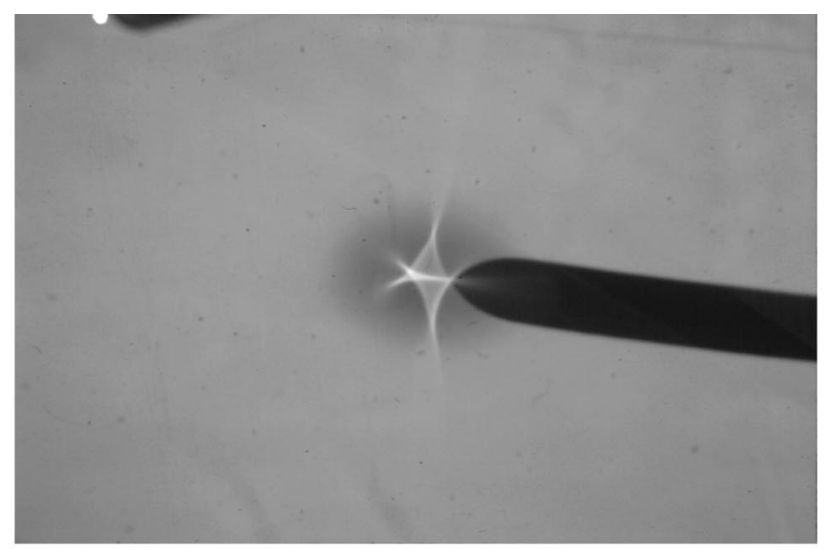

(b)

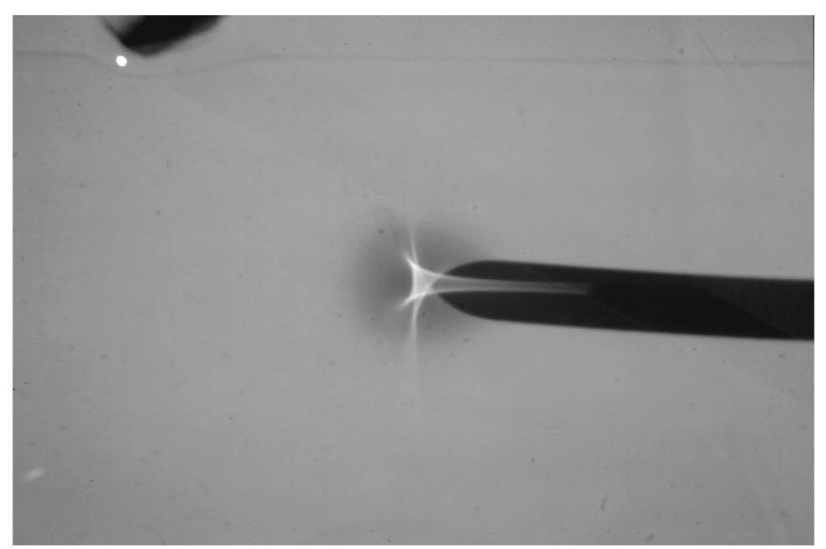

(d)

Fig. 2. Refraction caustic produced when a portion of the flat end of (a) a tree branch and (b)-(d) a 2.5-cm wooden dowel rod is raised above the water level and illuminated by the Sun. In (b)-(d) a progressively larger fraction of the flat end of the dowel rod lies above the water surface.

where $K_{p}(r / L)$ are modified Bessel functions and have the asymptotic behavior ${ }^{10}$

$$
\lim _{r \rightarrow \infty} \mathrm{K}_{p}(r / L)=(\pi L / 2 r)^{1 / 2} \exp (-r / L)
$$

If the rod extends vertically out of the liquid and the attachment of the liquid on the rod surface is a height $h$ above the $x y$ plane, the meniscus shape is independent of $\phi$, and in the small slope approximation is given by ${ }^{8,9}$

$$
f(r, \phi)=A_{0} K_{0}(r / L)
$$

where

$$
A_{0}=h / K_{0}(a / L) .
$$

If the rod is tilted from the vertical by the small angle $\gamma$ as in Fig. 3 so that the portion above the liquid points in the positive $x$ direction, we obtain an approximation to the meniscus shape using the following procedure. If a meniscus did not form around the rod and the liquid surface remained flat, the intersection of the rod with the liquid would be the ellipse

$$
x_{\text {rod }}^{2} \cos ^{2}(\gamma) / a^{2}+y_{\text {rod }}^{2} / a^{2}=1,
$$

or

$$
r_{\text {rod }}(\phi)=a /\left[1-\cos ^{2}(\phi) \sin ^{2}(\gamma)\right]^{1 / 2} .
$$

For this situation, symmetry considerations require $\delta_{p}=0$ in Eq. (4). The meniscus is observed to climb higher up the tilted rod along the positive $x$ axis where the angle between the rod and the asymptotic water surface is acute than along the negative $x$ axis where the angle is obtuse. As a result, we approximate the height of the meniscus on the rod surface as

$$
f\left[r_{\text {rod }}(\phi), \phi\right] \approx h[1+q \cos (\phi)],
$$

with $0<q \ll 1$. To determine the coefficients $\epsilon_{p}$ in Eq. (4) consistent with Eq. (10), we first expand $r_{\text {rod }}(\phi)$ in Eq. (9) for small $\gamma$ in terms of $\cos (p \phi)$ with the coefficient of each term being a series in powers of $\sin ^{2}(\gamma)$. Substituting this for $r$ in Eq. (4), Taylorseries expanding the modified Bessel functions about $a / L$ assuming $(a / L) \sin ^{2}(\gamma) \ll 1$, reexpanding the re- 


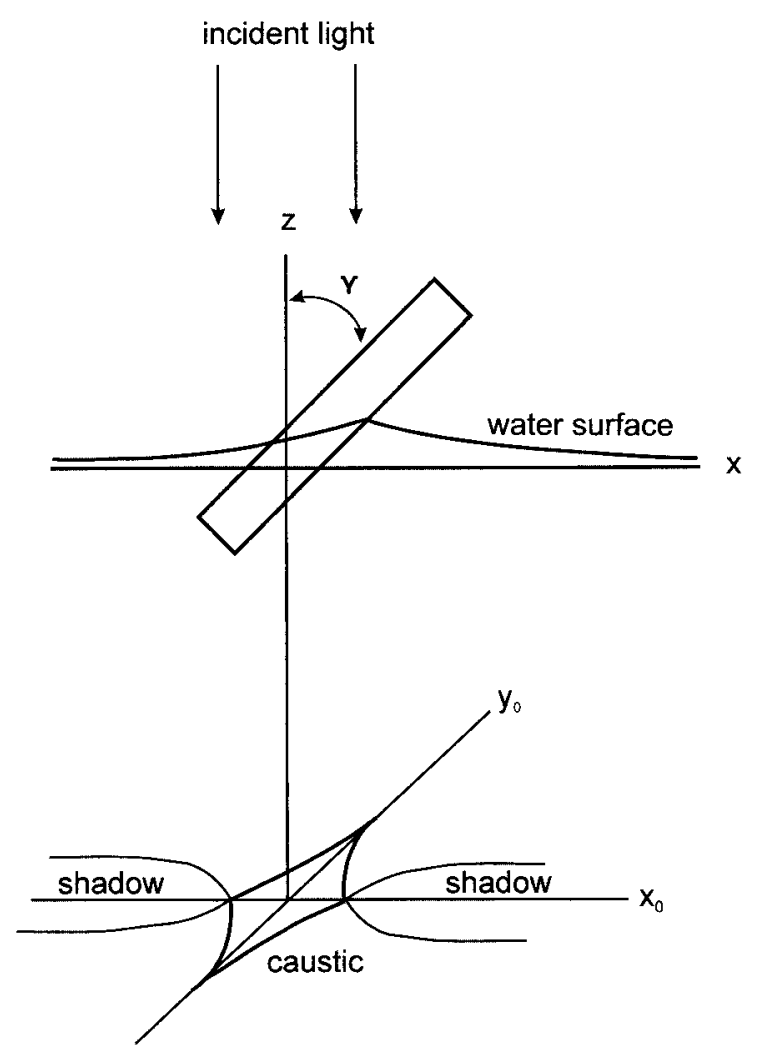

Fig. 3. Geometry of the shadow-sausage caustic for a tilted rod and vertically incident light.

sult in terms of $\cos (p \phi)$, and equating final sum to the approximate attachment profile of relation (10), we obtain

$$
\begin{aligned}
A_{0}= & h / K_{0}(a / L)+O\left[(a / L) \sin ^{2}(\gamma)\right], \\
\epsilon_{1}= & q K_{0}(a / L) / K_{1}(a / L)+O\left[q(a / L) \sin ^{2}(\gamma)\right], \\
\epsilon_{2}= & -(a / 4 L) \sin ^{2}(\gamma) K_{0}{ }^{\prime}(a / L) / K_{2}(a / L) \\
& +O\left[(a / L)^{2} \sin ^{4}(\gamma)\right] \\
\epsilon_{3}= & -(q a / 8 L) \sin ^{2}(\gamma) K_{0}(a / L) \\
& \times K_{1}{ }^{\prime}(a / L) /\left[K_{3}(a / L) K_{1}(a / L)\right] \\
& +O\left[q(a / L)^{2} \sin ^{4}(\gamma)\right] \\
\epsilon_{4}= & O\left[(a / L)^{2} \sin ^{4}(\gamma)\right] \\
\epsilon_{5}= & O\left[q(a / L)^{2} \sin ^{4}(\gamma)\right] \\
\epsilon_{6}= & O\left[(a / L)^{3} \sin ^{6}(\gamma)\right]
\end{aligned}
$$

and so on, where $K_{0}{ }^{\prime}$ is the derivative of $K_{0}$ with respect to its argument. Since $K_{0}$ is a decreasing function of $r / L, K_{0}{ }^{\prime}<0$ and $\epsilon_{2}>0$. As the rod is tilted from the vertical, the most important perturbations to the normal-incidence meniscus shape of Eqs. (6) and (7) are proportional to $\cos (\phi)$ because of the angle-dependent height of the liquid attachment to the rod surface and $\cos (2 \phi)$ because of the noncircular intersection of the rod with the liquid surface. As long as $(a / L) \sin ^{2}(\gamma) \ll 1$ and $q \ll 1$, the $\epsilon_{p}$ coef- ficients of Eq. (11) fall off rapidly as a function of $p$. Thus we neglect the $p \geq 3$ terms in Eq. (4).

\section{Shape of the Refraction Caustic}

\section{A. General Considerations}

Consider an electromagnetic plane wave propagating in the $-z$ direction and refracted into the liquid at the meniscus as in Fig. 3. Assuming that the slope of the liquid surface is everywhere small, the electric field at the coordinate

$$
\mathbf{r}_{0}=x_{0} \mathbf{u}_{x}+y_{0} \mathbf{u}_{y}=r_{0} \cos \left(\phi_{0}\right) \mathbf{u}_{x}+r_{0} \sin \left(\phi_{0}\right) \mathbf{u}_{y}
$$

on a viewing screen a distance $z_{0}$ beneath the liquid surface is given in the paraxial approximation by ${ }^{5,11}$

$$
E\left(\mathbf{r}_{0}, z_{0}\right)=\int_{-\infty}^{\infty} \mathrm{d} x \int_{-\infty}^{\infty} \mathrm{d} y \exp \left[i k F\left(\mathbf{r}, \mathbf{r}_{0}\right)\right]
$$

where $k=2 \pi / \lambda$ is the wave number of the incident light, $n$ is the refractive index of the liquid, and

$$
\begin{aligned}
F\left(\mathbf{r}, \mathbf{r}_{0}\right)= & (n-1) f(r, \phi)+n r^{2} /\left(2 z_{0}\right) \\
& -n r r_{0} \cos \left(\phi-\phi_{0}\right) / z_{0} .
\end{aligned}
$$

The first term of Eq. (14) is the optical path length from the raised meniscus to the $x y$ plane in Fig. 3, and the remaining two terms describe paraxial propagation in the liquid from the $x y$ plane to the $x_{0} y_{0}$ plane. In the short-wavelength limit, a light ray crossing the $x y$ plane at $\mathbf{r}$ arrives at $\mathbf{r}_{0}$ in the $x_{0} y_{0}$ plane where the relation between $\mathbf{r}$ and $\mathbf{r}_{0}$ is given by the stationary phase condition ${ }^{5,11}$

$$
\nabla F=0,
$$

where $\nabla$ contains derivatives with respect to $r$ and $\phi$. Substitution of Eq. (14) into Eq. (15) gives

$$
\begin{aligned}
x_{0}= & x+\left[(n-1) z_{0} / n\right][(\partial f / \partial r) \cos (\phi) \\
& -(\partial f / \partial \phi) \sin (\phi) / r], \\
y_{0}= & y+\left[(n-1) z_{0} / n\right][(\partial f / \partial r) \sin (\phi) \\
& +(\partial f / \partial \phi) \cos (\phi) / r] .
\end{aligned}
$$

A refraction caustic containing the point $\mathbf{r}_{0}$ in the $x_{0} y_{0}$ plane is formed when two or more neighboring rays crossing the $x y$ plane at $\mathbf{r}$ arrive together at $\mathbf{r}_{0}$ to produce focusing. The Gaussian curvature of the wave front vanishes at these caustic-producing points $\mathbf{r}$, i.e., $5,7,11$

$$
\begin{array}{r}
{\left[(n-1)\left(\partial^{2} f / \partial r^{2}\right)+n / z_{0}\right]\left[(n-1)(\partial f / \partial r) / r+n / z_{0}\right.} \\
\left.+(n-1)\left(\partial^{2} f / \partial \phi^{2}\right) / r^{2}\right]-(n-1)^{2}\left[\left(\partial^{2} f / \partial r \partial \phi\right)\right. \\
-(\partial f / \partial \phi) / r]^{2} / r^{2}=0 .
\end{array}
$$

The shape of the caustic in the $x_{0} y_{0}$ plane is obtained by substitution of the solution of Eq. (17) for $\mathbf{r}$ into Eqs. (16). 
B. Refraction Caustic for a Vertical Rod and Vertically Incident Light

For a circular cross-section rod standing vertically in the liquid and the meniscus shape of Eqs. (6) and (7), the solution of Eq. (17) is a circle of radius $R$ in the $x y$ plane where $R$ depends on $z_{0}$ by means of

$$
[(n-1) h /(n R L)]\left[K_{0}{ }^{\prime}(R / L) / K_{0}(a / L)\right]=-1 / z_{0} .
$$

The caustic in the $x_{0} y_{0}$ plane consists of the single point ${ }^{9}$

$$
x_{0}=y_{0}=0 .
$$

All the rays crossing the $x y$ plane on circles of different radii cross the $z$ axis together at different locations producing an axial caustic.

\section{Refraction Caustic for a Diagonal Rod and Vertically Incident Light}

A. Theory

The axial caustic of Eq. (19) is unstable with respect to perturbations of the meniscus shape as in Eq. (4). Substituting Eq. (4) into Eq. (17) and keeping terms to first order in the meniscus shape perturbation $\epsilon_{p}$ for $p \geq 1$, we obtain

$$
\begin{aligned}
0= & {[(n-1) h /(r L)]\left[K_{0}{ }^{\prime}(r / L) / K_{0}(a / L)\right] } \\
& +n / z_{0}+[(n-1) h /(r L)] \\
& \times \sum_{p=1}^{\infty} \epsilon_{p} U_{p}\left[K_{p}(r / L) / K_{0}(a / L)\right] \cos (p \phi),
\end{aligned}
$$

where

$$
U_{p}=K_{p}{ }^{\prime}(R / L)-\left(p^{2} L / R\right) K_{p}(R / L) .
$$

The solution $r(\phi)$ of Eq. (20) for $\epsilon_{p}=0$ is $r=R$, and the solution remains close to $R$ when the $\epsilon_{p}$ are small. Thus Taylor-series expanding $K_{0}{ }^{\prime}(r / L)$ about $R / L$ in Eq. (20), we obtain

$$
r(\phi)=R-L \sum_{p=1}^{\infty} \epsilon_{p} \cos (p \phi) U_{p} / W_{p},
$$

where

$$
W_{p}=K_{0}{ }^{\prime \prime}(R / L)-(L / R) K_{0}{ }^{\prime}(R / L) .
$$

Substitution of Eqs. (22) and (23) into Eqs. (16) gives the caustic shape 6 to first order in $\epsilon_{p}$ [indicated by the superscript (1)],

$$
\begin{aligned}
x_{0}^{(1)}= & {\left[(n-1) h z_{0} /(n R)\right] } \\
& \times \sum_{p=1}^{\infty} p \epsilon_{p}\left[K_{p}(R / L) / K_{0}(a / L)\right] g_{p}^{(x)}(\phi), \\
y_{0}^{(1)}= & {\left[(n-1) h z_{0} /(n R)\right] } \\
& \times \sum_{p=1}^{\infty} p \epsilon_{p}\left[K_{p}(R / L) / K_{0}(a / L)\right] g_{p}^{(y)}(\phi),
\end{aligned}
$$

where

$$
\begin{aligned}
& g_{p}^{(x)}(\phi)=p \cos (p \phi) \cos (\phi)+\sin (p \phi) \sin (\phi), \\
& g_{p}^{(y)}(\phi)=p \cos (p \phi) \sin (\phi)-\sin (p \phi) \cos (\phi) .
\end{aligned}
$$

For $p=1$, the caustic in the $x_{0} y_{0}$ plane remains a point focus translated off the $z$ axis to the location

$$
\begin{aligned}
& x_{0}^{(1)}=\left[(n-1) h z_{0} /(n R)\right] \epsilon_{1}\left[K_{1}(R / L) / K_{0}(a / L)\right], \\
& y_{0}^{(1)}=0 .
\end{aligned}
$$

For $p=2$, the caustic becomes the four-cusped astroid

$$
\begin{aligned}
x_{0}^{(1)}= & {\left[4(n-1) h z_{0} /(n R)\right] \epsilon_{2} } \\
& \times\left[K_{2}(R / L) / K_{0}(a / L)\right] \cos ^{3}(\phi), \\
y_{0}^{(1)}= & -\left[4(n-1) h z_{0} /(n R)\right] \epsilon_{2} \\
& \times\left[K_{2}(R / L) / K_{0}(a / L)\right] \sin ^{3}(\phi) .
\end{aligned}
$$

shown in Fig. 4(a). For $\epsilon_{2}>0$ as in Eq. (11), the cusps on the $\pm x_{0}$ axes are produced by rays crossing the $x y$ plane on the $\pm x$ axes. But the cusps on the $\pm y_{0}$ axes are produced by rays crossing the $x y$ plane on the $\mp y$ axes. These $\mp y$ rays refract toward the $z$ axis at the meniscus and cross over to produce the cusp reversal. The observed caustics of Fig. 1 exhibit a reversal of the $\pm y_{0}$ cusps as well, consistent with $\epsilon_{2}>0$ in Eqs. (11). Since the light rays crossing the $\pm x$ axes are blocked by the tree branch or wooden rod in Fig. 1, the corresponding cusps on the $\pm x_{0}$ axes do not appear. Although the astroid caustic studied in Ref. 6 in the context of glory scattering was a four-ray to two-ray transition, in this present context the astroid is a three-ray to one-ray transition, since far from the meniscus only one ray arrives at $\mathbf{r}_{0}$ after having been refracted at the asymptotically flat water surface. The diffraction structure apparent inside the astroid in Ref. 6 is not visible in Fig. 1 because the diffraction structure is exceedingly fine, since $\lambda \ll a$ and because the white light illuminating the wooden rod blurrs the diffraction pattern.

For $p=3$ the caustic becomes the three-cusped figure

$$
\begin{aligned}
x_{0}^{(1)}= & {\left[24(n-1) h z_{0} /(n R)\right] \epsilon_{3}\left[K_{3}(R / L) / K_{0}(a / L)\right] } \\
& \times\left[\cos ^{4}(\phi)-\cos ^{2}(\phi) / 2-1 / 8\right], \\
y_{0}^{(1)}= & -\left[24(n-1) h z_{0} /(n R)\right] \epsilon_{3}\left[K_{3}(R / L) / K_{0}(a / L)\right] \\
& \times \sin ^{3}(\phi) \cos (\phi),
\end{aligned}
$$

shown in Fig. 4(b). The three cusps occur at $\phi_{0}=0^{\circ}$, $120^{\circ}$, and $240^{\circ}$, and the entire caustic is traced out identically twice for $0 \leq \phi<2 \pi$.

In Eqs. (11) the $p=1$ meniscus shape perturbation to second order and $p=2$ perturbation to first order are both proportional to $\sin ^{2}(\gamma)$, and are thus potentially comparable in importance if $q$ is not small. The caustic of the $p=1$ perturbation taken to second order in $\epsilon_{p}$ [indicated by the superscript (2)] is obtained when we substitute Eq. (4) into Eq. (17), solve 


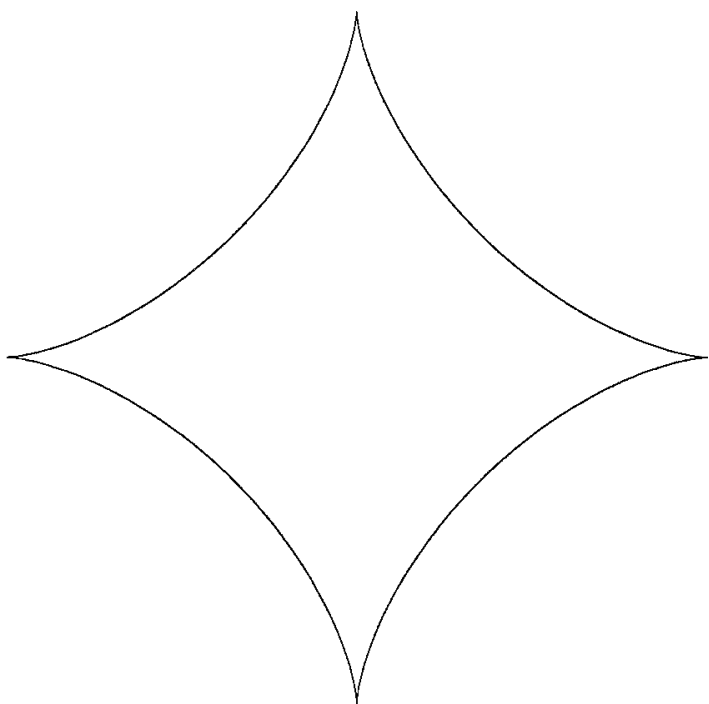

(a)

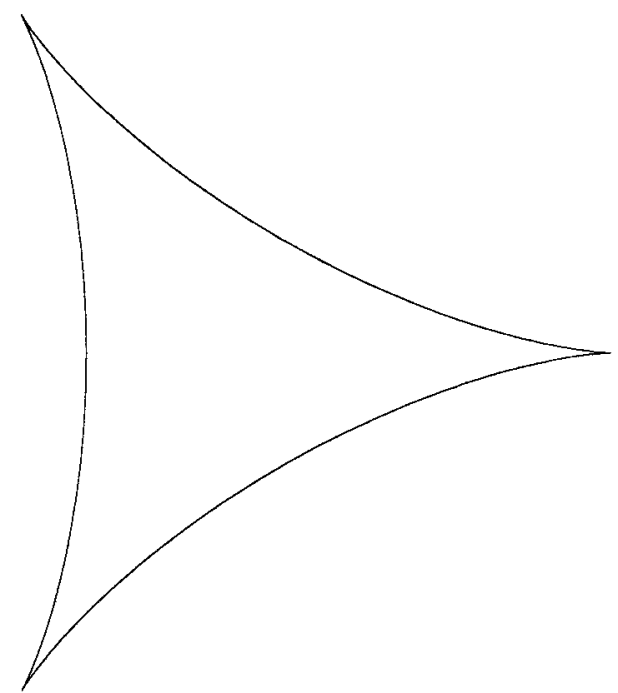

(b)

Fig. 4. Caustic shape to first order for (a) $p=2$ and (b) $p=3$. The $x_{0}$ axis is horizontal, and the $y_{0}$ axis is vertical.

Eq. (17) to second order in $\epsilon_{p}$, and substitute the result into Eqs. (16). We obtain

$$
\begin{aligned}
x_{0}^{(2)}= & x_{0}^{(1)}+\left[p(n-1) h z_{0} /(n R)\right] \epsilon_{p}{ }^{2} V_{p} \\
& \times\left[p V_{p} \sin ^{2}(p \phi) \cos (\phi)-U_{p}\right. \\
& \left.\times \cos (p \phi) g_{p}^{(x)}(\phi)\right] /\left[W_{p} K_{0}(a / L)\right], \\
y_{0}^{(2)}= & y_{0}^{(1)}+\left[p(n-1) h z_{0} /(n R)\right] \epsilon_{p}{ }^{2} V_{p} \\
& \times\left[p V_{p} \sin ^{2}(p \phi) \sin (\phi)-U_{p}\right. \\
& \left.\times \cos (p \phi) g_{p}^{(y)}(\phi)\right] /\left[W_{p} K_{0}(a / L)\right],
\end{aligned}
$$

where

$$
V_{p}=K_{p}{ }^{\prime}(R / L)-(L / R) K_{p}(r / L) .
$$

For $p=1$, Eqs. (29) become the translated astroid caustic

$$
\begin{aligned}
x_{0}^{(2)}= & {\left[(n-1) h z_{0} /(n R)\right]\left[\epsilon_{1} K_{1}(R / L)\right.} \\
& \left.-\epsilon_{1}^{2}\left(V_{1}^{2} / W_{1}\right) \cos ^{3}(\phi)\right] / K_{0}(a / L), \\
y_{0}^{(2)}= & {\left[(n-1) h z_{0} /(n R)\right] \epsilon_{1}^{2}\left(V_{1}^{2} / W_{1}\right) } \\
& \times \sin ^{3}(\phi) / K_{0}(a / L),
\end{aligned}
$$

which has the $\pm x_{0}$ cusps reversed rather than the $\pm y_{0}$ cusps. The fact that the $\pm y_{0}$ cusps are reversed in Fig. 1 indicates that for $a \gg L$ the $p=2$ contribution to the caustic to first order dominates over the $p=1$ contribution to second order. As a result, the height variation of the attachment of the liquid to the rod's surface satisfies

$$
q^{2}<(a / L) \sin ^{2}(\gamma)
$$

If it had turned out that $q^{2}>(a / L) \sin ^{2}(\gamma)$, the $\pm x_{0}$ cusps of the composite astroid would have been reversed, and if $q^{2}=(a / L) \sin ^{2}(\gamma)$, the $p=1$ contribution to second order and $p=2$ contribution to first order would have canceled, producing a translated point focus. An analogous effect occurs for the focusing caustic of a lens possessing coma, astigmatism, and spherical aberration ${ }^{12}$ and in the human eye. ${ }^{13}$

For $p \geq 2$, Eqs. (29) do not simplify further. But if the radius of the rod is much larger than the length scale of the meniscus, i.e., $a \gg L$, with the aid of Eq. (5) we obtain

$$
\begin{aligned}
x_{0}^{(2)} \approx & {\left[p(n-1) h z_{0} /(n R)\right](a / R)^{1 / 2} \exp [-(R-a) / L] } \\
& \times\left[\epsilon_{p} g_{p}^{(x)}(\phi)-\epsilon_{p}{ }^{2} g_{2 p}^{(x)}(\phi) / 2\right], \\
y_{0}^{(2)} \approx & {\left[p(n-1) h z_{0} /(n R)\right](a / R)^{1 / 2} \exp [-(R-a) / L] } \\
& \times\left[\epsilon_{p} g_{p}^{(y)}(\phi)-\epsilon_{p}{ }^{2} g_{2 p}^{(y)}(\phi) / 2\right] .
\end{aligned}
$$

The second-order correction to the caustic shape for the $p$ meniscus perturbation is identical to the shape of the caustic of the $2 p$ meniscus perturbation taken to first order. For $p=2$ and $0<\epsilon_{2}<0.2$ the astroid is only slightly deformed by the second-order correction. But for $\epsilon_{2}>0.2$, the $\pm x_{0}$ cusps point inward rather than outward and locally evolve into butterfly caustics $^{11}$ so as to connect to the $\pm y_{0}$ cusps, which continue to point outward. This is illustrated in Fig. 5 (a) for $\epsilon_{2}=0.5$. If we had $\epsilon_{2}<-0.2$ instead, the $\pm y_{0}$ cusps would have evolved into butterfly caustics while the $\pm x_{0}$ cusps would have been stable. For $p=3$ the second-order correction breaks the twofold degeneracy of the caustic and separates the cusps at $\phi_{0}=0^{\circ}, 120^{\circ}$, and $240^{\circ}$ into pairs of neighboring cusps as is illustrated in Fig. 5(b) for $\epsilon_{3}=0.3$. In addition, one cusp from each pair evolves into a butterfly caustic for $\epsilon_{3}>0.23$.

The position of the pointed ends of the two shadow segments in Fig. 1 is determined as follows. Since $q$ $\ll 1$, we approximate the shape of the liquid surface 


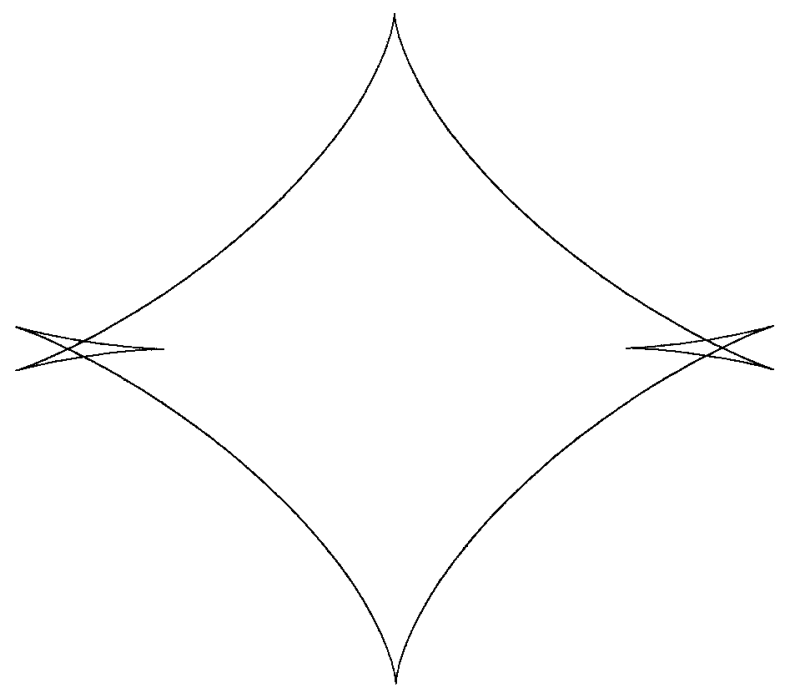

(a)

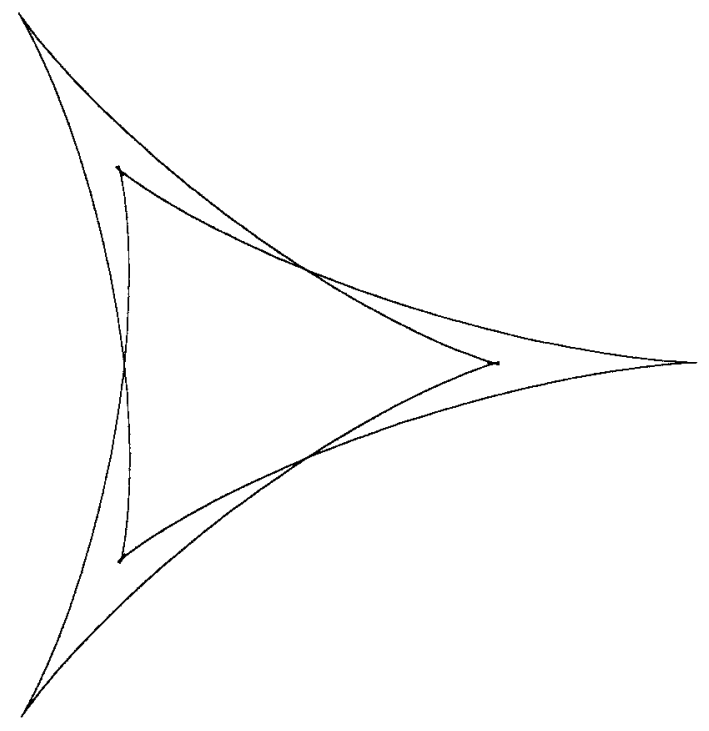

(b)

Fig. 5. Caustic shape to second order for (a) $p=2, \epsilon_{2}=0.5$ and (b) $p=3, \epsilon_{3}=0.3$. The $x_{0}$ axis is horizontal, and the $y_{0}$ axis is vertical.

by the $p=0,2$ terms of Eq. (4). Equations (16) then become

$$
\begin{aligned}
x_{0}= & x+x\left[(n-1) h z_{0} /(n r L)\right]\left[K_{0}{ }^{\prime}(r / L)+\epsilon_{2} K_{2}{ }^{\prime}(r / L)\right. \\
& \left.\times\left(x^{2}-y^{2}\right) / r^{2}+4 \epsilon_{2} K_{2}(r / L) L y^{2} / r^{3}\right] / K_{0}(a / L),
\end{aligned}
$$

$$
\begin{aligned}
y_{0}= & y+y\left[(n-1) h z_{0} /(n r L)\right]\left[K_{0}{ }^{\prime}(r / L)+\epsilon_{2} K_{2}{ }^{\prime}(r / L)\right. \\
& \left.\times\left(x^{2}-y^{2}\right) / r^{2}-4 \epsilon_{2} K_{2}(r / L) L x^{2} / r^{3}\right] / K_{0}(a / L),
\end{aligned}
$$

or, when we substitute Eq. (20) with $p=2$ into Eq. $(34 \mathrm{a})$,

$$
\begin{aligned}
x_{0}= & x y_{0} / y+x\left[4(n-1) h z_{0} /\left(n r^{2}\right)\right] \epsilon_{2} \\
& \times\left[K_{2}(r / L) / K_{0}(a / L)\right] .
\end{aligned}
$$

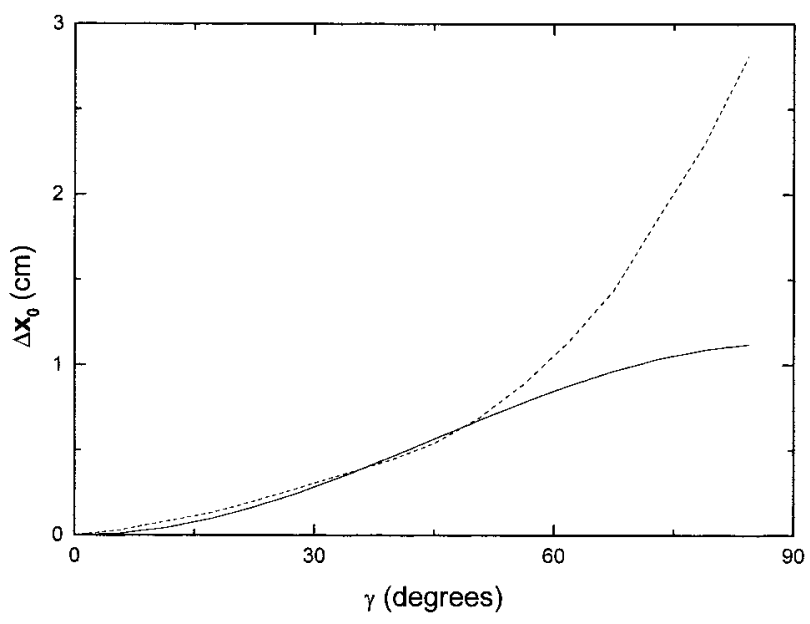

Fig. 6. Separation of the pointed shadow ends $\Delta x_{0}$ of a tilted rod for vertically incident light as a function of the rod tilt angle $\gamma$. The data of Fig. 7 of Ref. 1 form the dashed curve, and the leastsquares fit of Eq. (38) is the solid curve.

The edges of the shadow of the rod in the $x y$ plane occur at $y= \pm a$ for arbitrary $x$ as in Fig. 3, and the pointed end of the shadow segment occurs at $y_{0}=0$. The solution to Eq. (34b) for $\epsilon_{2} \rightarrow 0$ under these conditions is $r=R$, and when $\epsilon_{2} \ll 1$ the solution for $r$ remains close to $R$. Substituting $y_{0}=0$ and $r=R$ into Eq. (35), we obtain for the location of the pointed shadow ends

$$
\begin{aligned}
x_{0} \approx & \pm\left[4(n-1) h z_{0} / n\right] \epsilon_{2}\left[K_{2}(R / L) / K_{0}(a / L)\right] \\
& \times\left(R^{2}-a^{2}\right)^{1 / 2} / R^{2}+O\left(\epsilon^{2}\right) .
\end{aligned}
$$

The location in the $x_{0} y_{0}$ plane where the rod's shadow touches the astroid caustic is obtained by substitution of $y= \pm a$ into Eq. (27) to give

$$
\begin{aligned}
x_{0} \approx & \pm\left[4(n-1) h z_{0} / n\right] \epsilon_{2}\left[K_{2}(R / L) / K_{0}(a / L)\right] \\
& \times\left(R^{2}-a^{2}\right)^{3 / 2} / R^{4}, \\
y_{0} \approx & \mp\left[4(n-1) h z_{0} / n\right] \epsilon_{2}\left[K_{2}(R / L) / K_{0}(a / L)\right] a^{3} / R^{4} .
\end{aligned}
$$

Comparison of relations (36) and (37) shows that the penumbra of the rod's shadow begins at the connection to the astroid, whereas its umbra begins farther out at the pointed shadow ends. Finally, we obtain $x_{0} \rightarrow x$ and $y_{0} \rightarrow a$ in Eqs. (34) as $x \rightarrow \infty$ and $y=a$. The shadow of the rod's edges lies directly beneath the edges as the liquid surface becomes asymptotically flat.

\section{B. Experimental Confirmation}

As was seen in Eqs. (11), since $\epsilon_{2}$ is proportional to $\sin ^{2}(\gamma)$, the separation of the pointed shadow ends $\Delta x_{0}$ in relation (36) should also be proportional to $\sin ^{2}(\gamma)$. In Fig. 6 we show the least-squares fit of the function

$$
\Delta x_{0}=C \sin ^{2}(\gamma)
$$




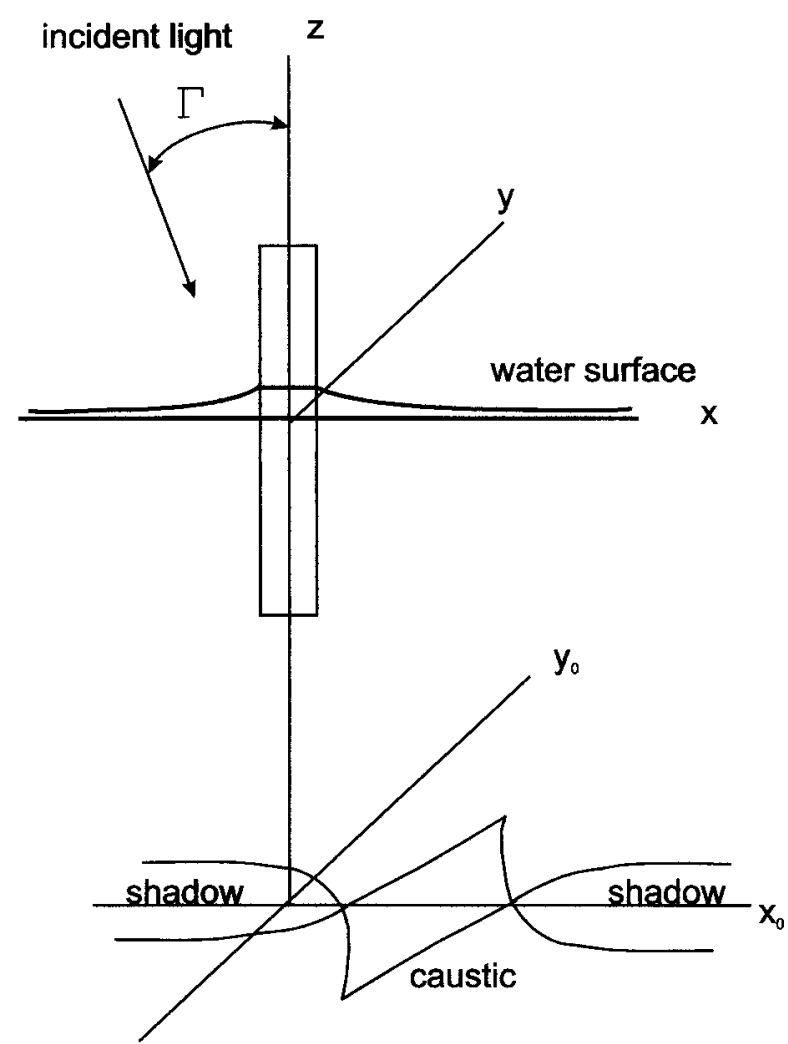

Fig. 7. Geometry of the shadow-sausage caustic for a vertical rod and diagonally incident light.

to the shadow end separation data of Ref. 1 Fig. 7, using $0^{\circ} \leq \gamma \leq 45^{\circ}$ for the fitting interval and subtracting off the baseline in the experimental data. The fit is reasonably good for $\gamma \lesssim 50^{\circ}$. For larger $\gamma$ the data rise at a much faster rate than the fit of Eq. (38), indicating the importance of more accurately modeling the meniscus shape for large rod tilt angles.

\section{Refraction Caustic for a Vertical Rod and Diagonally Incident Light}

\section{A. Theory}

We now consider a circular cross section rod standing vertically in the water with the source of incident light displaced from the vertical by the small angle $\Gamma$ in the $-x$ direction as in Fig. 7. The optical path length of the light rays from the surface of constant phase on the incident wave front containing the point $x=0, y=0, z=H$ to the $x y$ plane is found after much algebra to be

$$
\begin{aligned}
l(r, \phi)= & H\left(1-\Gamma^{2} / 2\right)+(n-1) h\left(1+\Gamma^{2} / 2 n\right) \\
& \times\left[K_{0}(r / L) / K_{0}(a / L)\right]-\left[(n-1)^{2} h^{3} /\left(2 n L^{2}\right)\right] \\
& \times\left[K_{0}(r / L) K_{0}^{\prime 2}(r / L) / K_{0}^{3}(a / L)\right] \\
& +r \Gamma \cos (\phi)+(n-1) N(r) \Gamma \cos (\phi) \\
& +(n-1) M(r) \Gamma^{2} \cos ^{2}(\phi)+O\left(\Gamma^{3}\right),
\end{aligned}
$$

where

$$
\begin{aligned}
N(r)= & -\left[h^{2} /(n L)\right]\left[K_{0}(r / L) K_{0}{ }^{\prime}(r / L) / K_{0}{ }^{2}(a / L)\right], \\
M(r)= & {\left[h^{3} /\left(2 n^{2} L^{2}\right)\right]\left[K_{0}{ }^{2}(r / L) K_{0}{ }^{\prime \prime}(r / L) / K_{0}{ }^{3}(a / L)\right] } \\
& +\left[\left(3+3 n-2 n^{2}\right) h^{3} /\left(4 n^{3} L^{2}\right)\right] \\
& \times\left[K_{0}(r / L) K_{0}{ }^{\prime 2}(r / L) / K_{0}{ }^{3}(a / L)\right],
\end{aligned}
$$

and the $L / R \ll 1$ assumption has been used to omit small corrections to the third and the final terms of Eq. (39). The $(n-1) N(r) \Gamma \cos (\phi)$ term in Eq. (39) is analogous to coma in the optical path length of skew rays passing through a spherical lens, ${ }^{12}$ and the $(n-$ 1) $M(r) \Gamma^{2} \cos ^{2}(\phi)$ term is analogous to astigmatism. Using $l(r, \phi)$ in place of $(n-1) f(r, \phi)$ in Eq. (14), we find that the coma term to first order in $\Gamma$ translates the point focus of Eq. (19) along the $x_{0}$ axis, the astigmatism term to first order in $\Gamma^{2}$ produces an astroid caustic with the $\pm y_{0}$ cusps reversed, and the coma term to second order in $\Gamma$ produces an astroid caustic with the $\pm x_{0}$ cusps reversed, i.e.,

$$
\begin{aligned}
x_{0}= & {\left[(n-1) z_{0} /(n R)\right][\Gamma N(R)} \\
& \left.+\left(T_{M}-T_{N}\right) \Gamma^{2} \cos ^{3}(\phi)\right], \\
y_{0}= & -\left[(n-1) z_{0} /(n R)\right]\left(T_{M}-T_{N}\right) \Gamma^{2} \sin ^{3}(\phi)
\end{aligned}
$$

with

$$
\begin{aligned}
T_{M}= & 2 M(R), \\
T_{N}= & \left(L^{2} / h\right)\left[N(R) / R-N^{\prime}(R)\right]^{2} K_{0}(a / L) /\left[K_{0}^{\prime \prime}(R / L)\right. \\
& \left.-(L / R) K_{0}{ }^{\prime}(R / L)\right] .
\end{aligned}
$$

Again assuming $R \gg L$ and substituting Eq. (5) into relations (33) and (34), we obtain

$$
\begin{aligned}
T_{M} \approx & {\left[h^{3} /\left(2 n^{3} L^{2}\right)\right]\left(3+5 n-2 n^{2}\right)(a / R)^{3 / 2} } \\
& \times \exp [-3(R-a) / L], \\
T_{N} \approx & {\left[4(n-1) h^{3} /\left(n^{2} L^{2}\right)\right](a / R)^{3 / 2} } \\
& \times \exp [-3(R-a) / L] .
\end{aligned}
$$

As was the case for the tilted rod in Section 4, if it were to happen that $T_{M}=T_{N}$, the contribution to the caustic shape of the two astroids would cancel and the caustic would remain a translated point focus. But for water with $n=1.33$, we obtain

$$
T_{M} \approx 1.72 T_{N},
$$

and the resulting astroid caustic has the $\pm y_{0}$ cusps reversed in agreement with the experimental observations of Fig. 1.

\section{B. Experimental Confirmation}

As a test of Eq. (42) we measured the separation of the cusp points $\Delta y_{0}$ when $\mathrm{He}-\mathrm{Ne}$ laser light, expanded with a ball lens, was diagonally incident on a 6.3-mm-diameter steel rod extending vertically out of a transparent tray filled with water. A sheet of graph paper was placed beneath the tray, and the 


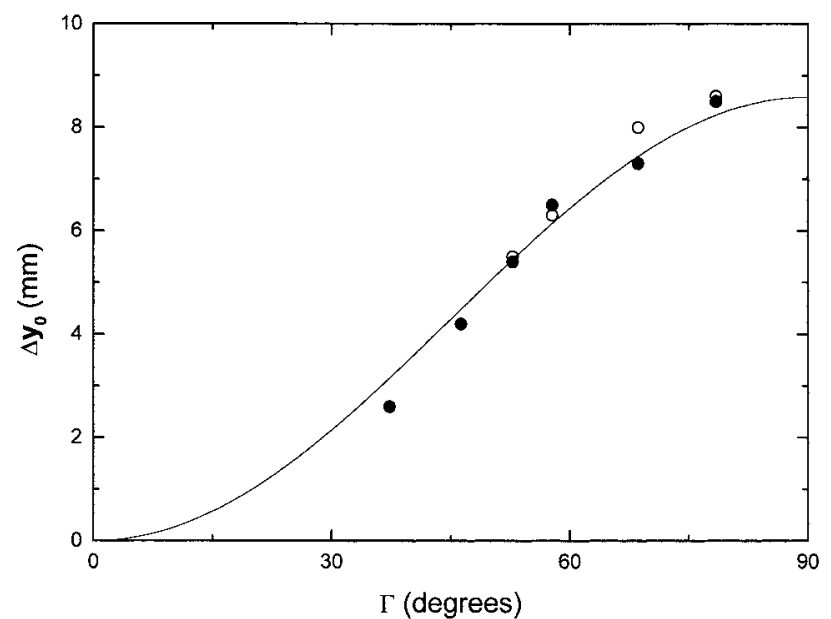

Fig. 8. Separation of the cusp points $\Delta y_{0}$ (filled circles) for a vertical rod and diagonally incident light as a function of the light's angle of incidence $\Gamma$. The least-squares fit of Eq. (48) to the data is the solid curve, and the results of a ray-tracing computer program are the open circles.

caustic pattern on the graph paper was recorded with a Nikon 990 digital camera equipped with a $3 \times$ zoom lens. The separation of the cusp points was measured on the digital camera image. The results, least-squares fitted to the equation

$$
\Delta y_{0}=C^{\prime} \sin ^{2}(\Gamma),
$$

are shown in Fig. 8. The comparison is quite good. In addition, we wrote a computer program tracing a family of diagonally incident rays through the meniscus to a viewing screen corresponding to the location of the graph paper in the experiment just described. The computer program did not employ the smallangle approximation assumed in the analytical calculations of Sections 4 and 5 and used the approximate large-slope meniscus shape derived in Ref. 1. The cusp point separation determined from the resulting spot diagram of ray intersections with the viewing screen is also shown in Fig. 8, and the fit to the data again is good.

\section{Refraction Caustic for Normally Incident Light with a Portion of the End of the Rod above the Liquid Surface}

As was seen in Section 2, if a curved meniscus did not form, the intersection of the liquid surface with a

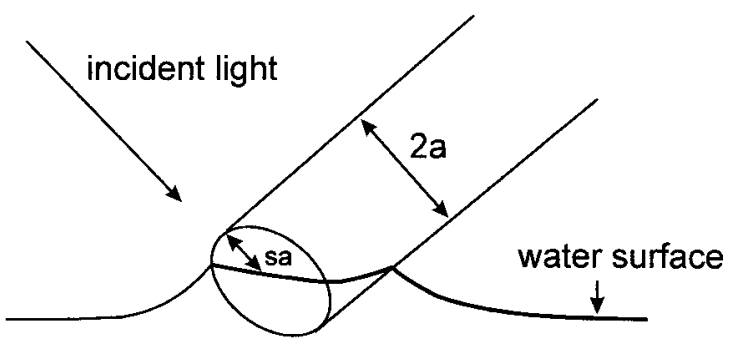

Fig. 9. Meniscus formation when a portion of the flat end of the rod is raised above the water surface. circular cross-section rod of radius $a$ tilted by the angle $\gamma$ would be an ellipse of semimajor axis $a / \cos (\gamma)$. Consider raising the tilted rod upward so that the water surface intersects the rod partially along its curved side and partially along its flat end as in Fig. 9. The meniscus now has a straight segment at its attachment to the flat end. Let the length of the flat end of the rod above the liquid surface be $s a$ where $0<s<1$. If a curved meniscus did not form, the intersection of the rod with the water is the truncated ellipse

$$
\begin{aligned}
r_{\mathrm{rod}}(\phi) & =a /\left[1-\cos ^{2}(\phi) \sin ^{2}(\gamma)\right]^{1 / 2} & & \text { if } 0 \leq \phi \leq \phi_{0}, \\
& =-a(1-s) /[\cos (\phi) \cos (\gamma)] & & \text { if } \phi_{0} \leq \phi \leq \pi,
\end{aligned}
$$

where $\pi / 2<\phi_{0}$ and

$$
\cos \left(\phi_{0}\right)=-(1-s) /\left[\cos ^{2}(\gamma)+(1-s)^{2} \sin ^{2}(\gamma)\right]^{1 / 2} .
$$

The Fourier-series decomposition of Eqs. (48) and (49) is

$$
r_{\text {rod }}(\phi)=\alpha_{0}+\sum_{p=1}^{\infty} \alpha_{p} \cos (p \phi)
$$

where

$$
\begin{aligned}
& \alpha_{0}=(1 / \pi) \int_{0}^{\pi} \mathrm{d} \phi r_{\text {rod }}(\phi), \\
& \alpha_{p}=(2 / \pi) \int_{0}^{\pi} \mathrm{d} \phi \cos (p \phi) r_{\text {rod }}(\phi) .
\end{aligned}
$$

The dependence of the Fourier coefficients $\alpha_{p}$ on $s$ for $1 \leq p \leq 5$ and for a representative tilt angle $\gamma=30^{\circ}$ is given in Fig. 10. For $s \gtrsim 0$ the dominant Fourier contribution to the truncated ellipse shape is $\alpha_{2}$, since the elliptical shape is encoded primarily in the $p=2$ Fourier coefficient. But for increasing $s, \alpha_{2}$ decreases in importance and the leading Fourier con-

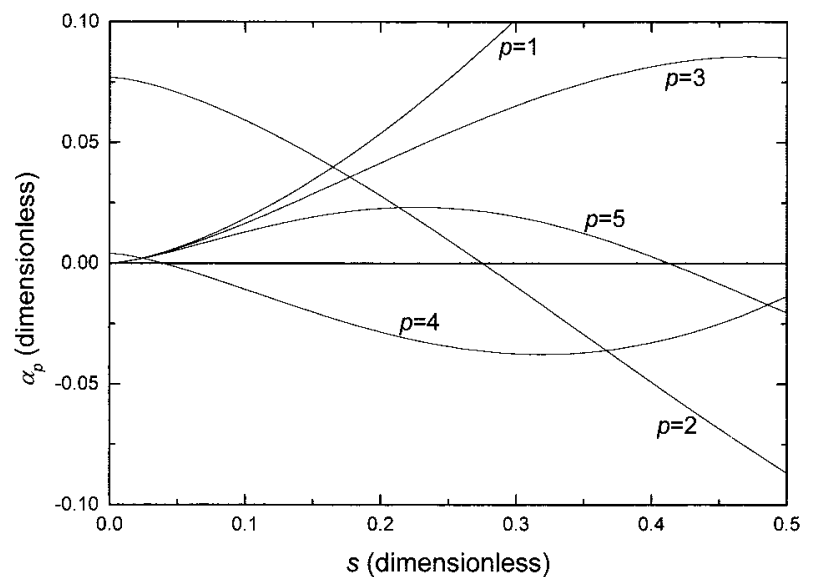

Fig. 10. $p=1,2,3,4,5$ Fourier coefficients $\alpha_{p}$ of a truncated ellipse as a function of the truncation fraction $s$ for a rod tilt angle of $\gamma=30^{\circ}$. 


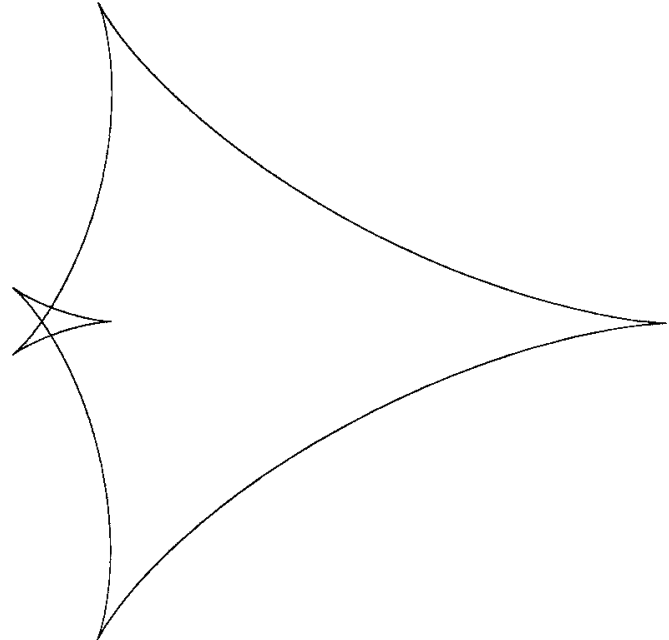

(a)

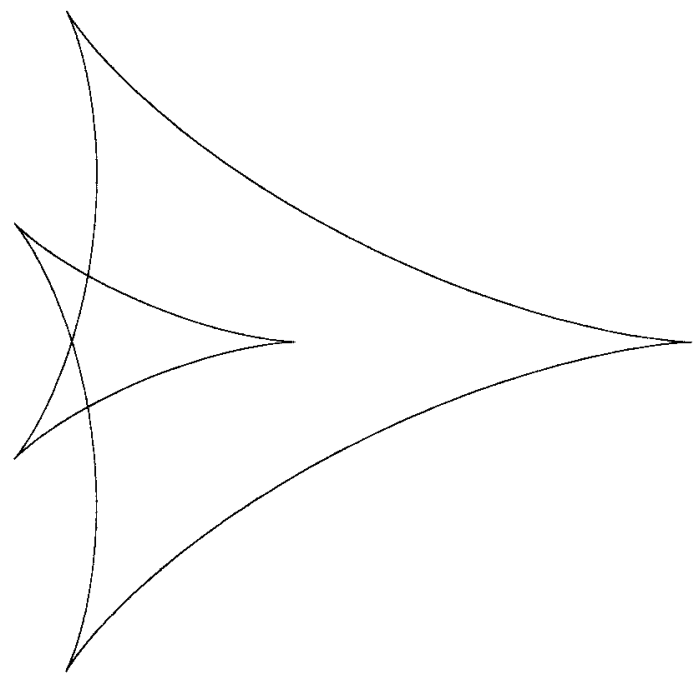

(b)

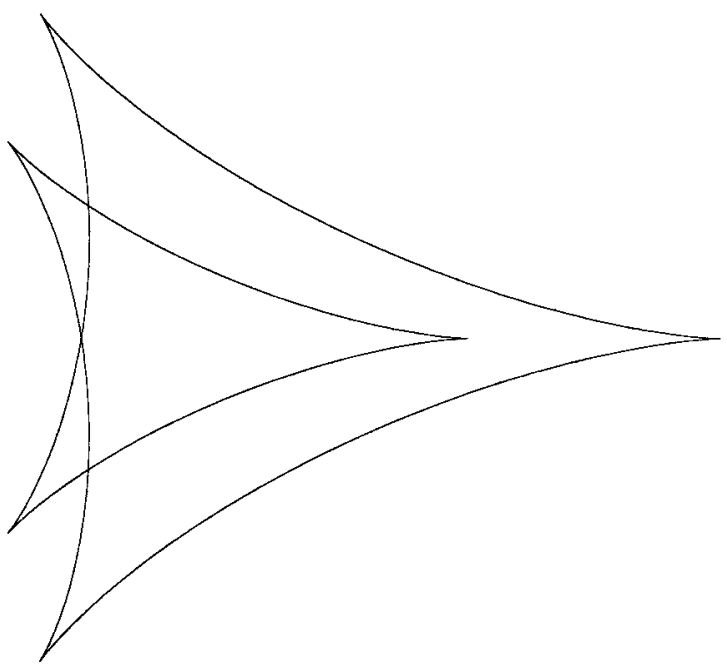

(c)

Fig. 11. Composite $p=2,3$ caustic of relation (54) for (a) $\epsilon_{2}: \epsilon_{3}=$ $2: 1$, (b) $\epsilon_{2}: \epsilon_{3}=1: 1$, and (c) $\epsilon_{2}: \epsilon_{3}=1: 2$. The $x_{0}$ axis is horizontal, and the $y_{0}$ axis is vertical. tributions to the truncated ellipse shape become $\alpha_{1}$ and $\alpha_{3}$, reflecting the growing importance of the straight segment. As was seen in Eqs. (9) and (11), the largest Fourier coefficients $\alpha_{p}$ of the shape of the water-rod intersection give rise to the largest meniscus shape coefficients $\epsilon_{p}$ in Eq. (4). The $p=1$ meniscus shape component merely translates the caustic produced by the combination of the $p=2,3$ meniscus shape components,

$$
\begin{aligned}
& x_{0} \approx \epsilon_{2} g_{2}^{(x)}(\phi)+\epsilon_{3} g_{3}^{(x)}(\phi), \\
& y_{0} \approx \epsilon_{2} g_{2}^{(y)}(\phi)+\epsilon_{3} g_{3}^{(y)}(\phi) .
\end{aligned}
$$

As a result, if almost none of the flat end of the rod extends out of the water and $s \geqslant 0$, the refraction caustic is a $p=2$ astroid accompanied by a small three-cusp contribution resulting from the $p=3$ meniscus shape perturbation. This was observed in Fig. 2(b) and is illustrated in Fig. 11(a) for $\epsilon_{2}$ and $\epsilon_{3}$ in relation (54) with the ratio $2: 1$. If more of the flat end is out of the water and $s$ is larger, the $p=2,3$ meniscus shape contributions are comparable, giving a caustic intermediate between the four-cusped astroid of Fig. 3(a) and the three-cusped figure of Fig. 3(b). The resulting caustic in Fig. 2(c) has five widely separated but interlocking cusps as does the illustration in Fig. 11(b) with $\epsilon_{2}: \epsilon_{3}=1: 1$ in relation (54). If yet more of the flat end is out of the water and $s$ continues to increase, the $p=3$ meniscus shape dominates and the $p=2$ contribution is only a small perturbation to it. The resulting caustic is a threecusped $p=3$ caustic with its twofold degeneracy only slightly broken by the $p=2$ perturbation, as is observed in Fig. 2(d) and illustrated in Fig. 11(c) for $\epsilon_{2}: \epsilon_{3}=1: 2$ in relation (54). The transition between the $p=2$ astroid caustic and the three-cusped $p=3$ caustic as a function of the ratio $\epsilon_{2}: \epsilon_{3}$ is as follows. First, the $-x_{0}$ cusp of the astroid evolves into a threecusped butterfly caustic. This then grows in size, and its three cusps approach and merge into the three remaining cusps of the astroid, producing the twofold-degenerate three-cusped $p=3$ caustic.

\section{Concluding Remarks}

As was mentioned in Section 1, the observations of Figs. 1 and 2 correspond to a rod tilted substantially from the vertical, sunlight incident on the meniscus substantially away from the vertical, and the meniscus slope not everywhere small. These specific observational conditions were not addressed in Sections 2-6 where the rod tilt was assumed to be small, the light was nearly vertically incident, the meniscus slope was everywhere small, the rod was substantially larger than the meniscus scale length, and the effects of rod tilt and diagonally incident light were considered individually rather than together. In spite of these idealizations, we successfully reproduced all the major features of the caustics of Figs. 1 and 2. This provides evidence that the mechanisms of caustic production discussed in Sections 2-6 continue to be qualitatively valid for larger rod tilts, 
farther off-axis incident beams, and larger surface slopes.

This research was supported in part by National Science Foundation grant \#PHY-9987862.

\section{References}

1. C. Adler, "Shadow-sausage effect," Am. J. Phys. 35, 774-776 (1967).

2. M. J. Smith, "Comment on: shadow-sausage effect," Am. J. Phys. 36, 912-914 (1968).

3. J. Walker, The Flying Circus of Physics with Answers (Wiley, New York, 1977), topic 5.3, pp. 115, 266.

4. J. Walker, "Shadows cast on the bottom of a pool are not like other shadows. Why?" Sci. Am. 259(1), 116-119 (1988).

5. M. V. Berry, "Waves and Thom's theorem," Adv. Phys. 25, 1-26 (1976).

6. W. P. Arnott and P. L. Marston, "Unfolding axial caustics of glory scattering with harmonic angular perturbations of toroidal wave fronts," J. Acoust. Soc. Am. 85, 1427-1440 (1989).

7. P. L. Marston, "Geometrical and catastrophe optics methods in scattering," Phys. Acoust. 21, 1-234 (1992), pp. 220-221.

8. C. Huh and L. E. Scriven, "Shapes of axisymmetric fluid interfaces of unbound extent," J. Colloid. Interface Sci. 30, 323337 (1969).

9. M. V. Berry and J. V. Hajnal, "The shadows of floating objects and dissipating vortices," Opt. Acta 30, 23-40 (1983).

10. G. Arfken, Mathematical Methods for Physicists, 3rd ed. (Academic, New York, 1985), p. 618, Eq. (11.127).

11. M. V. Berry and C. Upstill, "Catastrophe optics: morphologies of caustics and their diffraction patterns," Prog. Opt. 18, 257-346 (1980), Sect. 3, pp. 277-297.

12. Ref. 11, Appen. 2, pp. 339-342.

13. H. Hofer and D. R. Williams, "The eye's mechanisms for autocalibration," Opt. Photon. News (January 2002), pp. 3439. 